\title{
Editorial: Endocrine Forms of Hypertension: Clinical and Emerging Molecular Aspects
}

\author{
Peter Igaz ${ }^{1,2+}$ and Teresa Maria Seccia ${ }^{3 * \dagger}$ \\ ${ }^{1}$ Second Department of Internal Medicine, Faculty of Medicine, Semmelweis University, Budapest, Hungary, ${ }^{2}$ MTA-SE \\ Molecular Medicine Research Group, Hungarian Academy of Sciences, Semmelweis University, Budapest, Hungary, ${ }^{3}$ Clinica \\ dell'Ipertensione Arteriosa, Department of Medicine, University of Padua, Padua, Italy
}

Keywords: hypertension, primary aldosteronism, thyroid, licorice, Cushing's syndrome, microRNA

\section{Editorial on the Research Topic}

\section{Endocrine Forms of Hypertension: Clinical and Emerging Molecular Aspects}

Secondary hypertension is responsible for about 5-20\% of all hypertension cases depending on the examined cohorts (1-3). In contrast with primary essential hypertension that can only be treated, secondary hypertension often manifesting itself as resistant hypertension includes potentially curable diseases (1-3). Research on endocrine diseases leading to secondary hypertension is a progressive field, and better elucidating their pathophysiology and clinical features, might pave the way for novel diagnostic modalities and treatments. The major endocrine diseases as causes of hypertension include primary aldosteronism, hypercortisolism, pheochromocytoma/paraganglioma, thyroid diseases, primary hyperparathyroidism,

Edited and reviewed by: Pierre De Meyts,

Université Catholique de

Louvain, Belgium

${ }^{*}$ Correspondence:

Teresa Maria Seccia

teresamaria.seccia@unipd.it

tThese authors have contributed equally to this work

Specialty section:

This article was submitted to Molecular and Structural Endocrinology,

a section of the journa

Frontiers in Endocrinology

Received: 21 November 2019 Accepted: 22 November 2019 Published: 06 December 2019

Citation:

Igaz P and Seccia TM (2019) Editorial: Endocrine Forms of Hypertension: Clinical and Emerging Molecular Aspects. Front. Endocrinol. 10:857. doi: 10.3389/fendo.2019.00857 and acromegaly.

In this Research Topic, we have compiled several articles on different issues of endocrine secondary hypertension regarding both molecular and clinical features. Primary aldosteronism is the most common endocrine cause of secondary hypertension affecting $5-13 \%$ of hypertensive patients in different studies (4-6). Three articles are dedicated to its field in this Research Topic.

The study by Mohideen et al. was aimed at establishing the prevalence of KCNJ5 gene mutations in APAs from a Malaysian cohort. The authors, by examining 54 APAs from a Malaysian cohort, found that its prevalence was $31 \%$, similarly as reported in Caucasians, however, with no prevalence of mutants in females. KCNJ5 mutant and wild type APAs showed similar percentages of ZF- and ZG- like cells, but KCNJ5 mutant APAs with a ZF-like profile tended to be associated with larger APA. A higher expression of CYP11B2 was found in females, who also had adrenalectomy at a younger age than males. An interesting hypothesis was provided by the Authors: since females had often larger tumors, the phenotype previously associated with KCNJ5 mutant APAs could be the phenotype of APAs from female patients, not the phenotype of all KCNJ5 mutant APAs.

In the original study by Decmann et al., circulating microRNAs belonging to the group of non-coding RNA molecules $(7,8)$ are investigated as potential novel diagnostic tools for the differentiation of the two major forms of primary aldosteronism i.e., bilateral adrenal hyperplasia and unilateral adenoma. Three microRNAs were validated on a large cohort of samples to be overexpressed in bilateral hyperplasia relative to the unilateral adenoma samples. Although sensitivity and specificity values were not found to be high enough for clinical introduction at present, this study presents a novel class of potential diagnostic markers for differentiating subclasses of endocrine hypertension, but also underlines that primary aldosteronism could be regarded as a spectrum disease. 
A comprehensive review on licorice-induced pseudohyperaldosteronism is given by Sabbadin et al., who discussed its biochemical picture and the mechanisms underlying the mineralocorticoid effect. Of interest, they also discuss the potential therapeutic use of licorice related to its anti-androgen and estrogen-like activity, mostly exploited for treatment of polycystic ovary syndrome on top of spironolactone, and the anti-inflammatory effects of licorice, unveiling unfamiliar properties of an old medicinal plant.

The pathophysiology and treatment of hypertension in Cushing's syndrome is discussed in the mini review by Barbot et al.. Several relevant molecular mechanisms are presented, and most notably antihypertensive and also cortisol-lowering treatments are discussed. Clinically relevant issues are also discussed in the mini review by Canu et al. where the hypertension-related clinical picture,

\section{REFERENCES}

1. Calhoun DA, Nishizaka MK, Zaman MA, Thakkar RB, Weissmann P. Hyperaldosteronism among black and white subjects with resistant hypertension. Hypertension. (2002) 40:8926. doi: 10.1161/01.hyp.0000040261.30455.b6

2. Rossi GP, Seccia TM, Pessina AC. Secondary hypertension: the ways of management. Curr Vasc Pharmacol. (2010) 8:75368. doi: 10.2174/157016110793563843

3. Wei FF, Zhang ZY, Huang QF, Staessen JA. Diagnosis and management of resistant hypertension: state of the art. Nat Rev Nephrol. (2018) 14:42841. doi: 10.1038/s41581-018-0006-6

4. Rossi GP, Bernini G, Caliumi C, Desideri G, Fabris B, Ferri $\mathrm{C}$, et al. A prospective study of the prevalence of primary aldosteronism in 1,125 hypertensive patients. J Am Coll Cardiol. (2006) 48:2293-300. doi: 10.1016/j.jacc.2006.07.059

5. Käyser SC, Dekkers T, Groenewoud HJ, Van Der Wilt GJ, Carel Bakx J, Van Der Wel MC, et al. Study heterogeneity and estimation of prevalence of primary aldosteronism: a systematic review and meta-regression analysis. JClin Endocrinol Metab. (2016) 101:2826-35. doi: 10.1210/jc.2016-1472 genetics and treatment issues of pheochromocytoma are presented.

Thyroid diseases are very frequent, but they less often affect blood pressure values. A comprehensive view of the effects of thyroid hormones on the cardiovascular system is provided by Berta et al., who also discuss the genetic background that may favor cardiovascular damage.

We hope that the reader will find this Research Topic interesting, and the molecular and clinical issues covered by the articles will be helpful in both research and clinical management of secondary endocrine hypertension.

\section{AUTHOR CONTRIBUTIONS}

Both authors listed have made a substantial, direct and intellectual contribution to the work, and approved it for publication.

6. Monticone S, D'Ascenzo F, Moretti C, Williams TA, Veglio F, Gaita F, et al. Cardiovascular events and target organ damage in primary aldosteronism compared with essential hypertension: a systematic review and meta-analysis. Lancet Diabetes Endocrinol. (2018) 6:41-50. doi: 10.1016/S2213-8587(17)30319-4

7. Igaz I, Igaz P. Tumor surveillance by circulating microRNAs: a hypothesis. Cell Mol Life Sci. (2014) 71:4081-7. doi: 10.1007/s00018-014-1682-4

8. Igaz P. Circulating microRNAs in adrenal tumors. Curr Opin Endocrinol Diabetes Obes. (2019) 26:155-9. doi: 10.1097/MED.0000000000000472

Conflict of Interest: The authors declare that the research was conducted in the absence of any commercial or financial relationships that could be construed as a potential conflict of interest.

Copyright (C) 2019 Igaz and Seccia. This is an open-access article distributed under the terms of the Creative Commons Attribution License (CC BY). The use, distribution or reproduction in other forums is permitted, provided the original author(s) and the copyright owner(s) are credited and that the original publication in this journal is cited, in accordance with accepted academic practice. No use, distribution or reproduction is permitted which does not comply with these terms. 